\title{
Study on the Correlation between job adaptation obstacle and perceived social support of community nurses in Changchun
}

\author{
Wei Meng, Yujin Liua ${ }^{a}$, Wenjing Sun, Xiufeng Zhao, Fengge Dong, Guixiu Yan, Bing Yu and \\ Zhuang Cong \\ Welfare College of Nursing of College of Humanities\&Northeast Normal University, 130117, Changchun, China
}

\begin{abstract}
Objective: To investigate the present situation of job adaptation and perceived social support of community nurses in Changchun, and to explore the relevance between them, for the purpose of providing the basis for community nursing managers to implement effective human resource management. Methods: A general demographic information questionnaire, job adaptation obstacle scale and perceived social support scale were used to investigate 290 community nurses in Changchun. Results: The score of job adaptation obstacle was $20.85 \pm 5.18$; the score of perceived social support was $64.25 \pm 10.32$, the score of support in the family was $20.01 \pm 3.58$, and the score of the support out of family was $42.57 \pm 6.86$; the job adaptation obstacle was negatively correlated with the perceived social support, the support in the family, and the support out of family. Conclusion: The job adaptation situation of the nurses in the survey communities was generally poor and the perceived social support was at a moderate level. Therefore, community nursing managers should actively understand the situation of nurse job adaptation, and then take effective measures to improve the community nurses social support, improve the current situation of the poor job adaptation of the community nurses, and prevent loss of nursing talents, for the improvement of the quality of nursing service.
\end{abstract}

\section{Introduction}

In recent years, with the progress and development of community medical service in China, the community nurse attracts more and more people's attention. Foreign studies on community nurses are the issues of the job satisfaction and work motivation, job stress and job burnout, work environment and safety, et al[1-3]. Domestic studies on community nurses mainly involve the occupational values, job involvement, job stress and job satisfaction and so on[4-7].The above studies have revealed that the nurse job adaptation affects the quality of nursing service, and a poor nursing job adaptation is inclined to causing a series of problems, such as the lowered work enthusiasm, the tense relationship between nurses and patients, and declined quality of nursing service[8].The concept of perceived social support was put forward by Ausubel first as early as 1958, which has caught the attention of scholars in recent years and has been widely used in various fields[9]. At present, the research about

\footnotetext{
${ }^{a}$ Corresponding author: Liu Yujin, E-mail:1257819211@qq.com

This work was supported by a Social Sciences planning project of Department of Education of Jilin Province (2014).
} 
the situation of community nurse job adaptation and perceived social support is fewer in China, and moreover, there is fewer research on the correlation between job adaptation obstacles and perceived social support in nurses. Therefore, this study is aimed at understanding the present situation of community nurses job adaptation and perceived social support through the survey of community nurses, and exploring the correlation between them, which may provide references for the management of the community nursing human resources, thereby stabilizing the community nursing teams, improving the quality of community nursing service, and promoting the progress and development of community nursing career.

\section{Research content}

\subsection{Research object}

By using the method of cluster sampling, a questionnaire survey was carried among nurses of the health service centres (stations) of five districts (Chaoyang District, Kuancheng District, Luyuan District, Erdao District, and Nanguan District) in Changchun. Inclusion criteria: (1) community registered nurses; (2) Nurses with Chinese nurse qualification certificate; (3) those who volunteered to fill in the questionnaires and participate in the survey. Exclusion criteria: (1) Community nursing interns;(2) Those who were not on duty due to going out for study, pregnancy and puerperium during the survey.

\subsection{Research methods}

\subsubsection{Survey method}

A questionnaire survey was used and there were three questionnaires. (1) General demographic information questionnaire, including age, cultural level, professional rank, etc.; (2) Job adaptation obstacle scale: The job adaptation obstacle scale Wa compiled by Tyollaska[10] was adopted, the scale could reflect a series of individuals' views and interests in reality and their own motivation. The job adaptation obstacle scale in Chinese version should have a good reliability and validity. In this research, the job adaptation obstacle scale' Cronbach's alpha was 0.78 . The scale included 37 items, in which answering "yes" was scored 1 point for 29 items and answering "no" was scored 1 point for eight items, its total scores were 37 points, and the higher the score, the more the lack of work motivation and the maladaptation. (3) Perceived social support scale (PSSS): The scale compiled by Zimet,et al in 1987 was used[11].The scale was translated and amended by Chinese scholars Jiang Qianjin[12] in 2001, and the revised scale with a good reliability and validity has been widely used in China. Self-perception is emphasized in PSSS, and what is assessed with the scale is the support degree that an individual can perceive in the different social relations. There were 12 items in the scale, which was divided into two dimensions, namely the support in the family (parents, relatives, and spouse, etc.) and the support out of family (colleagues, leaders, friends, etc.). The score of support in the family included the cumulative scores of item 3,4,8, and 11, and the score of support out of family was made up of cumulative score of the other items. Each item was evaluated using a sevenlevel scoring method (Likert scale), which meant that the higher the score, the more the perceived social support. 300 Survey questionnaires were given out and 290 valid questionnaires were recovered, with a valid response rate of $96.7 \%$.

\subsubsection{Statistical method}

Statistical Package for the Social Sciences (SPSS) 21.0 for Windows was used to perform the statistical analyses. Percentage was used to describe count data, $\mathrm{X} \pm \mathrm{S}$ was used to describe measurement data, Pearson correlation analysis was used to measure the correlation between job 
adaptation obstacle and perceived social support, and a significance level of 0.05 or lower was considered statistically significant.

\section{Results}

\subsection{General demographic information}

Nurses' age was more concentrated in 25 to 29 years old, the majority had 2-5 years of working years, nurses with the professional title of nurse accounted for $70.7 \%$, the number of them in a contract system of employment form accounted for $56.9 \%$, and most of them had the college degree and were unmarried (see Table 1).

Table 1. General demographic information (\%)

\begin{tabular}{|c|c|c|c|}
\hline Variables & Groups & $\mathbf{n}$ & $\mathbf{( \% )}$ \\
\hline Age(year) & $\leq 24$ & 70 & 24.1 \\
\hline & $25-29$ & 160 & 55.2 \\
\hline & $30-34$ & 31 & 10.7 \\
\hline & $\geq 35$ & 29 & 10.0 \\
\hline Working year(year) & $\leq 1$ & 70 & 24.1 \\
\hline & $2-5$ & 130 & 44.8 \\
\hline & $6-10$ & 43 & 14.8 \\
\hline & $\geq 11$ & 47 & 16.2 \\
\hline Marital status & unmarried & 150 & 51.7 \\
\hline & married & 138 & 47.6 \\
\hline & divorce & 2 & 0.7 \\
\hline Educational level & technical education & 44 & 15.2 \\
\hline & associate degree & 198 & 68.3 \\
\hline & bachelor's degree & 48 & 16.5 \\
\hline Technical title & junior RN & 205 & 70.7 \\
\hline & senior RN & 50 & 17.2 \\
\hline & nurse in charge & 30 & 10.3 \\
\hline & associate professor nurse & 5 & 1.7 \\
\hline & formal-employed nurses & 105 & 36.2 \\
\hline Employment form & contract-employed nurses & 165 & 56.9 \\
\hline & others & 20 & 6.9 \\
\hline
\end{tabular}

\subsection{Job adaptation situation}

The results showed that the nurses' job adaptation obstacle score was $20.85 \pm 5.18$, significantly higher than the Chinese female norm $17.28 \pm 5.14$ reported by Ji Shumao[13] with a statistically significant difference $(p<0.05)$.In addition, the results also showed that score differences in the job adaptation obstacle among the nurses with different educational levels, technical title, and employment form were statistically significant. The results of pairwise comparison showed that the job adaptation situation of nurses with bachelor's degree was worse than that of nurses with technical education and associate degree, that of nurse-in-charge and associate professor nurse was better than that of junior $\mathrm{RN}$ and senior RN, and that of contract-employed nurses was worse than that of formal-employed nurses, as shown in Table 2.

Table 2. Job adaptation situation $(\mathrm{n}=290)$

\begin{tabular}{|c|c|c|c|c|}
\hline Variable & Groups & job adaptation obstacle & F & P \\
\hline Age (year) & $\leq 24$ & $20.56 \pm 5.59$ & 1.386 & 0.709 \\
\hline & $25-29$ & $20.82 \pm 6.22$ & & \\
\hline & $30-34$ & $20.44 \pm 6.07$ & & \\
\hline
\end{tabular}




\begin{tabular}{|c|c|c|c|c|}
\hline & $\geq 35$ & $19.11 \pm 5.56$ & & \\
\hline Working year (year) & $\leq 1$ & $20.13 \pm 5.80$ & 5.641 & 0.131 \\
\hline & $2-5$ & $20.01 \pm 6.35$ & & \\
\hline & $6-10$ & $20.65 \pm 4.68$ & & \\
\hline & $\geq 11$ & $20.78 \pm 6.31$ & & \\
\hline Marital status & unmarried & $20.75 \pm 6.30$ & 2.703 & 0.259 \\
\hline & married & $21.02 \pm 5.57$ & & \\
\hline & divorce & $19.50 \pm 10.61$ & & 0.000 \\
\hline Educational level & technical education & $19.00 \pm 4.08$ & 16.875 & \\
\hline & associate degree & $19.73 \pm 4.33$ & & 0.015 \\
\hline & bachelor's degree & $22.22 \pm 6.54$ & & \\
\hline Technical title & junior RN & $22.96 \pm 5.09$ & 10.410 & \\
\hline & senior RN & $22.63 \pm 7.07$ & & \\
\hline & nurse in charge & $16.33 \pm 5.52$ & & \\
\hline & associate professor nurse & $15.50 \pm 3.62$ & & \\
\hline Employment form & formal-employed nurses & $18.50 \pm 3.21$ & 7.577 & \\
\hline & contract-employed nurses & $23.03 \pm 4.08$ & & \\
\hline & others & $22.29 \pm 3.27$ & & \\
\hline
\end{tabular}

\subsection{Perceived social support}

The total score of community nurses' perceived social support was $64.25 \pm 10.32$, the total score of family support was $20.01 \pm 3.58$, and the total score of support out of family was $42.57 \pm 6.86$.

\subsection{Correlation between job adaptation obstacle and perceived social support}

The results showed that there was a negative correlation between job adaptation obstacle and perceived social support $(\mathrm{p}<0.01)$. The detailed results are shown in Table 3 .

Table 3. Correlation between job adaptation obstacle and perceived social support (r-values)

\begin{tabular}{|c|c|}
\hline & Job adaptation obstacle \\
\hline Total perceived social support & -0.325 \\
\hline Support in family & -0.357 \\
\hline Support out of family & -0.261 \\
\hline
\end{tabular}

\section{Discussion}

\subsection{Analysis on the present situation of job adaptation obstacle of community nurses in Changchun}

The results showed that the score of job adaptation obstacle of community nurses in Changchun was $20.85 \pm 5.18$, significantly higher than the Chinese female norm $17.28 \pm 5.14$ reported by Ji Shumao, it indicated that the community nurses might lack the work motivation and be poor in the job adaptation. The reasons may be related to the community nurses' single working mode, fuzzy self-positioning, and low professional identity. Studies have revealed that community nurses' wages are lower, social benefits are relatively poor, social status is not high, but their working time is longer, workload is heavier, and they often engage in trivial work[14]. All these likely lead to the lack of work motivation, reduced work enthusiasm, lower job satisfaction, and even job adaptation obstacle gradually in community nurses[15]. Studies have also showed that the low level of wages is one of the important reasons for the turnover of community nurses, which can hinder the development of community nursing[14]. Therefore, the relevant departments should improve the community nurses' payment and stabilize the community nursing team. 
In addition, this research revealed that the job adaptation situation of nurses with bachelor's degree was worse than that with technical education and associate degree, which may be due to the reason that the nurses with bachelor's degree had a high expectation of future career in school, but after working in the community, they found that the gap between the actual work situation and the expectation was bigger, which lead to their apparent psychological imbalance and the generation of their contradicted emotion about the profession of community nurses, so it is easy for them to adapt their job poorly[16]. Studies have showed that the expectation of nursing profession of nurses with technical education and associate degree was lower, and when they join in work after graduation, they find that the gap between the actual work situation and expectation is not so significant, so that their psychological gap feeling is not so strong, and they are easy to produce the love of nursing work and have the work motivation, which may be the reason why the nurses with technical education and associate degree are not easy to appear job adaptation obstacle[17]. The job adaptation situation of nurse-in-charge and associate professor nurse was better than that of junior $\mathrm{RN}$ and senior $\mathrm{RN}$, which is different from the conclusion of clinical nurses' job adaptation obstacle made by Shao Xiaozhen[18]. The key reasons may be related to the following two aspects: (1) The investigation groups are different, and there are some differences in the group characteristics; (2) There is a certain gap in their working environment, working intensity, working pressure, etc. between clinical nurses and community nurses. The conclusion made in this study might be related to the shorter working time of junior RN' and senior RN', the lack of work experience and the ability to adapt themselves to changing circumstances, and the incapacity to realize their self-value when they engage in the community nursing profession, etc. The job adaptation situation of contract-employed nurses was worse than that of the formal-employed nurses, which might be because community contractemployed nurses' social benefits, chances of promotion, and further education outside were worse than formal-employed nurses'. All of them easily result in the lack of work motivation and the job adaptation obstacle in the community contract-employed nurses. Due to these reasons, community managers should pay attention to the mind and body change of community nurses, especially nurses with bachelor's degree, those working for shorter time, junior RN and senior RN, and contractemployed nurses, and take effective measures positively to promote community nurses to adapt the community health service work.

\subsection{Analysis on the present situation of community nurses' perceived social support in Changchun}

Perceived social support focuses on the emotional experience and (or) satisfaction of individual subjective feeling about understood, respected and supported[19].The results showed that the community nurses' perceived social support was at a moderate level, and the score of support out of family was higher, indicating that when the nurses had difficulties and needed help, the community colleagues, leaders, and friends gave them much support. On the one hand, it might be linked to most of nurses' time distributed on work, more contact with the colleagues and leaders than their families, in which the colleagues and leaders tended to be the first people to offer them help when problems occurred; on the other hand, it might be linked to the nurses likeness to pour out their thoughts with their friends, no intention to bring negative emotions to their families. Therefore, more care and help given to the nurses by community managers will help to improve the community nurses' perceived social support.

\subsection{Correlation between job adaptation obstacle and perceived social support of community nurses in Changchun}

This research results revealed that the job adaptation obstacle was negatively correlated with the perceived social support, support in the family, and support out of family, namely the lower perceived social support, the worse community nurses' job adaptation obstacle. For this reason, community nurses' families should properly give nurses understanding and support. Community nursing managers 
should often focus on the nurses' psychological change and work adaptation situation, and when they have emotional problems, managers should listen to nurses patiently and provide emotional support for them[20]; when nurses have work difficulties, nursing managers should give them guidance and help positively to enhance their self-confidence; the relevant departments should properly improve the payment of community nurses, give them more opportunities of further education and advanced studies [21], arouse the nurses' initiative to study and work, arouse their working enthusiasm, improve their sense of professional worth, and prevent their job burnout, all of which may contribute to improve nurses' perceived social support degree[22], thereby promoting community nurses to adapt to community nursing work, preventing the loss of community nurses, improving the quality of community nursing service, and promoting the progress and development of community nursing career.

\section{References}

1. Sacks E, Alva S, Magalona S,et al. Examining domains of community health nurse satisfaction and motivation: results from a mixed-methods baseline evaluation in rural Ghana. Human resources for health,13(2015).

2. McTiernan K, McDonald N. Occupational stressors, burnout and coping strategies between hospital and community psychiatric nurses in a Dublin region. Journal of psychiatric and mental health nursing,22,3(2015).

3. Terry D,Le, Q,Nguyen, $U$ et al. Workplace health and safety issues among community nurses: a study regarding the impact on providing care to rural consumers. BMJ open,5,8(2015).

4. Xu Ping, Zhu Lei, Lv Zhuohong et al. Community nurses' professional values and its influencing factors. Nursing research,30,5B(2016).

5. Wang Ruifang. Study on present situation and relationship between working environment and job involvement of community nurses. Disease surveillance and control,10,3(2016).

6. Guo Yan, Jiang Wenhui, Li Xiaomei, et al. Research about community nurses' job stressors influence on job satisfaction in Xi'an. Nursing management journal,16,1(2016).

7. Zheng Dan. Research progress of community nurses' job satisfaction. Nursing research,29, 1B(2015).

8. Xie Lixia, Quan Suqin, Jia Hongli, et al. Present situation research about nurses job adaptation obstacle in one Three-grade hospital in Hengyang. Chinese nursing research,9,3(2015).

9. Xiao Shuiyuan. The influence of social support to health of body and mind. Chinese journal of mental health,1,4 (1987).

10. Zhang Haiwei. Status of job adaptation obstacle and its influencing factors of male nurses. Chinese nursing research,25,9(2011).

11. Blumenthal JA, Burg MM, Barefoot J, et al. Social support, type A behavior, and coronaryartery disease. Psychosomatic Medicine,49(1987).

12. Huang Li, Jiang Qianjin, Ren Weihong. Correlation research of coping styles, social support, and the psychosomatic symptoms in cancer patients. Chinese journal of mental health,10,4(1996).

13. Ji Maoshu. Minnesota multiphase personality inventory, the latest research and multi-class scale explanation. Beijing:science press, 1(2004).

14. Yang Rongfang, Ma Jinghua, Wang Hui, et al. Current situation survey about nursing human resources of community health service center in Shijiazhuang. Journal of community medical journal,14,5(2016).

15. Hu Haixia, Zhang Minmin, Gao Yuxia. Correlation between the professional values and job burnout status of community nurse in part of community in Changchun. Journal of liberation army nursing,29,2B(2012).

16. Lu Qinrui, Wang Yanli, Zhao Xiaohong, et al. Current investigation about clinical nurses' professional attitudes and influencing factors. Chinese nursing research,3,30(2016).

17. Lu Xuya, Zhu Xiuwen. Nursing undergraduate professional attitudes influence factors analysis. Education of traditional Chinese medicine,24,1(2005). 
18. Shao Xiaozhen, Wang Cong. Correlation research between nurse' job adaptation obstacle and personality characteristics. Human resources management, 9 (2013).

19. Li Yingying. Relationship research about Junior high school students' gratitude and perceived social support, family functioning. Changchun: the Northeast Normal University(2013).

20. Zhou Haiping, Ma bo. Correlation research between alexithymia and perceived social support of ICU nurses. The nurse education magazine, 31,4 (2016).

21. Xin Li. Current analysis of community nurses. Traditional Chinese medicine in Inner Mongolia, 18(2012).

22. Zhou Zhili, Zhang Hui. Relationship between job burnout and perceived social support of nurses. Journal of occupational and health,27,13 (2011). 